REFER EN C E

1 Richey, M. W. (1975). The navigation of small craft. This Journal, 28, 477.

\title{
D. H. Sadler comments
}

THE idea of an 'Altitude-Azimuth' almanac is not new though most effort (see this Journal, Vol. I, I 85, 1948; Vol. 2, 285, 1949) has been concerned with the Sun and planets rather than the stars. For the stars the simple problem is that observations are recorded in mean time (say G.M.T.) whereas the positions of the stars depend on sidereal time (L.H.A. Aries) given by: L.H.A. Aries = G.H.A. Aries + west longitude. A full account of star tables (and diagrams) using a mean-time argument (and thus avoiding using the Almanac to give G.H.A. Aries) is given in 'The Genesis of the E.A.N.T.s' (this Journal, Vol. 6, 333, 1953). Ignoring the complications arising from the motion of the observer between sights, and corrections for precession and nutation, there are innumerable methods of combining

(a) the observed mean time;

(b) a 'day' or 'year' correction to give the time-argument of the tables;

(c) corrections to altitude for non-tabular arguments ;

(d) interpolation in the tables;

(e) plotting from a fixed position, or from one whose longitude is chosen to avoid interpolation.

For world-wide use the choice depends on the planned usage and on the relative merits of simplicity in arithmetic or in plotting, as well as with material considerations of the size and cost of the tables.

The tables described above relate to a small range of longitude only and, taking advantage of the limited duration of twilight, can use G.M.T. directly as argument. This may not be the most economical method, but it is certainly simple for the user. The brief description does not include a discussion of the effect of the possible use of large intercepts, of the possible errors in refraction for low altitudes, and the incorporation of corrections for precession and nutation. These should be examined if the method is extended to larger areas and the tables are used for many years.

\section{A Position Line from an Object off the Chart}

\author{
Captain A. B Moody U.S.N.R.
}

THE use of the traverse table for plotting a bearing from an object outside the coverage area of the chart, as described by Pesi Sorab, 1 suggests a graphical solution that might be preferred by some navigators. The method is analogous to advancing a position line by advancing the position of the object observed.

Refer to Fig. 1. The bearing of an object off the northern limit of the chart is observed. Any convenient point on the chart is selected as the position of the fictitious object, and the reciprocal of the measured bearing is plotted from this 